\title{
An operative gamma camera for sentinel lymph node procedure in case of breast cancer
}

\author{
S. Salvador, ${ }^{t}$ V. Bekaert, ${ }^{a}$ C. Mathelin, ${ }^{b}$ J.-L. Guyonnet ${ }^{a}$ and D. Huss ${ }^{a}$ \\ ${ }^{a}$ Institut Pluridisciplinaire Hubert Curien, UMR 7178 - CNRS/IN2P3 and ULP. BP 28, \\ 23 rue du Loess, 67037 Strasbourg Cedex 02, France \\ ${ }^{b}$ CHRU, Service de Gynécologie-Obstétrique, Hôpital Civil, \\ 1 place de l'Hôpital, 67091 Strasbourg Cedex, France \\ E-mail: samuel.salvadordires.in2p3.fr
}

\begin{abstract}
Large field of view gamma cameras are widely used to perform lymphoscintigraphy in the sentinel lymph nodes (SLN) procedure in case of breast cancer. However, they are not specified for this application and their sizes do not enable their use in the operative room to control the excision of the all SLN. We present the results obtained with a prototype of a new mini gamma camera developed especially for the operative lymphoscintigraphy of the axillary area in case of breast cancer. This prototype is composed of $10 \mathrm{~mm}$ thick parallel lead collimator, a $2 \mathrm{~mm}$ thick GSO:Ce inorganic scintillating crystal from Hitachi and a Hamamatsu H8500 flat panel multianode (64 channels) photomultiplier tube (MAPMT) equipped with a dedicated electronics. Its actual field of view is $50 \times 50 \mathrm{~mm}^{2}$. The gamma interaction position in the GSO scintillating plate is obtained by calculating the center of gravity of the fired MAPMT channels. The measurements performed with this prototype demonstrate the usefulness of this mini gamma camera for the pre, per and post-operative identification of SLN's and how its complementary role with an intraoperative handheld gamma probe enables to improve the efficiency of this practice. A $100 \times 100 \mathrm{~mm}^{2}$ field of view camera designated to cover the entire axillary area is under investigation.
\end{abstract}

KEYWORDS: Gamma camera, SPECT, PET PET/CT, coronary CT angiography (CTA); Medical-image reconstruction methods and algorithms, computer-aided so .

\footnotetext{
* Corresponding author.
} 


\section{Contents}

\begin{tabular}{|c|c|}
\hline 1. Introduction & $\mathbf{1}$ \\
\hline 2. Materials and methods & 2 \\
\hline 2.1 The collimator & 2 \\
\hline 2.2 Inorganic scintillating crystal & 3 \\
\hline 2.3 The multianode photomultiplier tube & 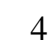 \\
\hline 2.4 The electronics & 4 \\
\hline 3. Calibration & 5 \\
\hline 3.1 Energy calibration & $\overline{5}$ \\
\hline 3.2 The image & 7 \\
\hline 3.3 Linearity & 8 \\
\hline .4 Uniformity & $\overline{9}$ \\
\hline
\end{tabular}

\begin{tabular}{|lr|}
\hline 4. Phantom study & 10 \\
\hline
\end{tabular}

\begin{tabular}{ll|}
\hline 5. Conclusions and perspectives & 11 \\
\hline
\end{tabular}

\section{Introduction}

The sentinel lymph node (SLN) procedure consists in the localization, excision and pathological examination of the first nodes to which lymphatic drainage from epithelial cancer cells occurs. The pathological analysis allows determining if the SLNs are cleaned or not of metastasis cells. At the present, the detection of SLNs is performed using a periareolar or peritumoral injection of a radiotracer (a colloidal suspension of 15 to $25 \mathrm{MBq}$ of ${ }^{99 \mathrm{~m}} \mathrm{Tc}$ emitting $\gamma$-rays of $140 \mathrm{keV}$ energy), the day before surgery or, sometimes, the day of the surgery. A lymphoscintigraphic image, made by the Nuclear Medicine Department allows counting the SLNs number. During surgery, the search of SLN is done by an intraoperative handheld gamma probe. Under controlled study conditions, the SLN procedure proved to be a reliable method for the evaluation of the axillary nodal status in patients with early-stage invasive breast cancer [1].

The use of an intraoperative gamma camera could accelerate the SLNs localization thanks to pre and peroperative lymphoscintigraphic images and control, with a postoperative image, the complete axillary clearance. In order to improve SLN detection, some intraoperative gamma cameras were tested. They are based on two classes of materials, semi conductors: zinc tellure $(\mathrm{CdZnTe})$ [2] [4], cadmium tellure (CdTe) [5], or inorganic long decay time scintillating crystal: sodium doped cerium iodide (CsI:Na) [6] tallium doped sodium iodide (NaI:Tl) [7]. Most of these devices have a too small field of view to map the entire axillary area. In addition, the choice of semi conductor as photodetector reaches a poor detection efficiency leading a long acquisition time. 


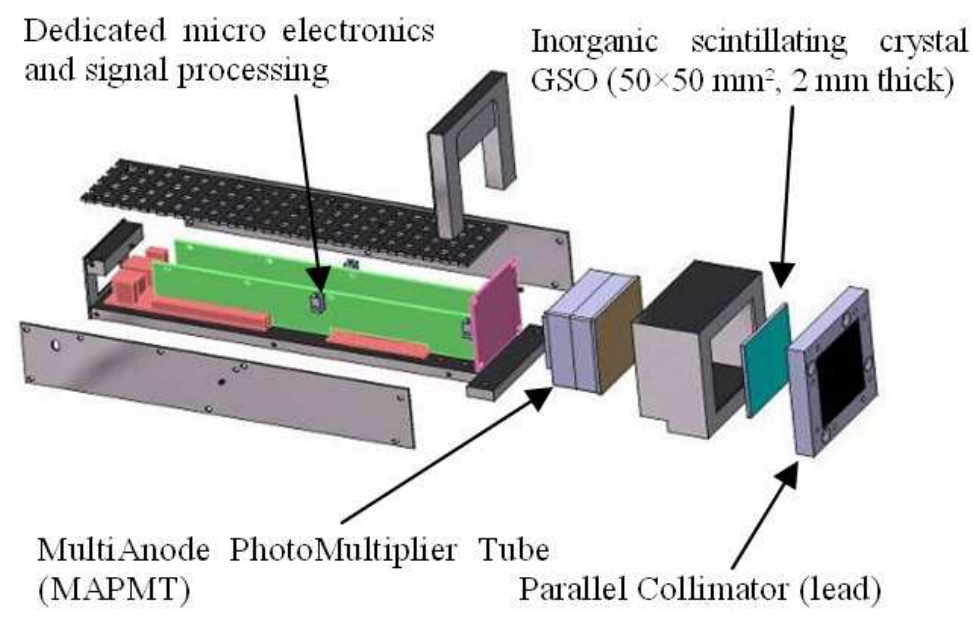

Figure 1. Design of the mini Gamma Camera prototype.

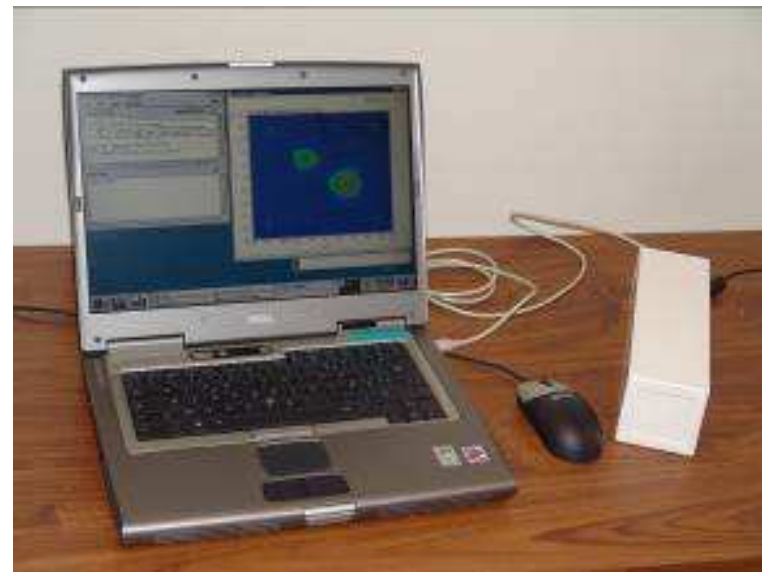

Figure 2. Picture of the mini Gamma Camera prototype with its associated computer.

The Institut Pluridiscipplinaire Hubert Curien (IPHC) developed an operative gamma camera named CarolIReS based on a fast decay time inorganic scintillating crystal allowing a good sensitivity and a short image acquisition time. This device is an additional tool to help the surgeon in the search of SLNs and will not replace the intraoperative probe [8]

\section{Materials and methods}

The studied prototype of the mini gamma camera is composed of a parallel lead collimator, a single fast decay time inorganic scintillating crystal, a multi channel photomultiplier tube, a fully dedicated electronics and a software treatment for the image building (figure 1). The field of view (FOV) of the device is $50 \times 50 \mathrm{~mm}^{2}$ and represents one quarter of the surface of the final mini gamma camera design. Figure 1 shows a picture of the mini gamma camera prototype connected to the PC needed for the software treatment and imaging.

\subsection{The collimator}

The gamma camera is equipped with a $10 \mathrm{~mm}$ thick parallel lead collimator weighting $360 \mathrm{~g}$. The hole diameter is $2.0 \mathrm{~mm}$ and the septa size is $0.2 \mathrm{~mm}$. The collimator features have been chosen in order to find a good compromise between spatial resolution and detection efficiency. 


\begin{tabular}{|c|c|c|c|c|c|c|}
\hline & $\begin{array}{l}\mathrm{GSO} \\
\mathrm{Gd}_{2} \mathrm{SiO}_{5}: \mathrm{Ce}\end{array}$ & $\begin{array}{l}\text { YAP } \\
\mathrm{YAlO}_{3}: \mathrm{Ce}\end{array}$ & $\begin{array}{l}\text { BrilLanCe }{ }^{\mathrm{TM}} 380 \\
\mathrm{LaBr}_{3}: \mathrm{Ce}\end{array}$ & $\begin{array}{l}\text { BrilLanCe }{ }^{\mathrm{TM}} 350 \\
\mathrm{LaCl}_{3}: \mathrm{Ce}\end{array}$ & $\begin{array}{l}\mathrm{LSO} \\
\mathrm{Lu}_{2} \mathrm{SiO}_{5}: \mathrm{Ce}\end{array}$ & $\begin{array}{l}\text { PreLude }{ }^{\mathrm{TM}} 420 \\
\mathrm{Lu}_{1.8} \mathrm{Y}_{0.2} \mathrm{SiO}_{5}: \mathrm{Ce}\end{array}$ \\
\hline$\rho\left(\mathrm{g} \cdot \mathrm{cm}^{-3}\right)$ & 6.71 & 5.37 & 5.29 & 3.79 & 6.50 & 7.10 \\
\hline$\mu / \rho\left(\mathrm{cm}^{2} \cdot \mathrm{g}^{-1}\right)$ & 0.854 & 0.319 & 0.652 & 0.704 & 1.250 & 1.442 \\
\hline $1 / \mu(\mathrm{cm})$ & 0.17 & 0.58 & 0.29 & 0.37 & 0.18 & 0.10 \\
\hline$Z_{\text {eff }}$ & 58 & 36 & 47 & 46 & 65 & 65 \\
\hline $\mathrm{n}$ & 1.85 & 1.95 & 1.88 & 1.90 & 1.82 & 1.81 \\
\hline$\tau(\mathrm{ns})$ & 56 & 25 & 26 & 28 & 40 & 41 \\
\hline$\lambda(\mathrm{nm})$ & 430 & 370 & 380 & 350 & 420 & 430 \\
\hline $\begin{array}{l}\text { Light Yield } \\
\left(\text { photons.MeV }{ }^{-1} \text { ) }\right.\end{array}$ & 9000 & 18000 & 63000 & 49000 & 30000 & 32000 \\
\hline Hygroscopic & no & no & yes & yes & no & no \\
\hline \multicolumn{4}{|c|}{$\begin{array}{l}\rho: \text { density } \\
\mu: \text { linear attenuation coefficient at } 140 \mathrm{keV} \\
\mu / \rho: \text { mass attenuation coefficient at } 140 \mathrm{keV} \\
\mathrm{Z}_{\text {eff: }} \text { effective } \mathrm{Z} \text { number }\end{array}$} & \multicolumn{3}{|c|}{$\begin{array}{l}\mathrm{n}: \text { refractive index } \\
\tau: \text { decay time constant } \\
\lambda: \text { Scintillation wavelength at maximum } \\
\quad \text { emission }\end{array}$} \\
\hline
\end{tabular}

Table 1. Features of different fast decay inorganic scintillating crystals.

The spatial resolution $R_{c}$ of a parallel collimator and its geometrical efficiency $\varepsilon_{c}$ are given by the formulas:

$$
\begin{gathered}
R_{c}=\frac{(d \cdot e)}{H_{e}} \\
\varepsilon_{c}=\frac{\sqrt{3}}{8 \pi}\left(\frac{e^{2}}{H_{e} \cdot(e+s)}\right)^{2}
\end{gathered}
$$

where $d$ is the distance between the source and the entrance surface of the collimator, $H_{e}$ the effective thickness of the collimator defined as $H_{e}=\mathrm{H}-2 / \mu\left(\mu=2.28 \mathrm{~mm}^{-1}\right.$ is the lead linear attenuation coefficient for $140 \mathrm{keV}$ gamma rays and $\mathrm{H}$ is the real thickness of the collimator), $e$ the hole diameter and $s$ the septa dimension (thickness of the material between the holes). The three geometrical parameters $e, H$ and $s$ have been calculated to obtain the best compromise between $R_{c}$ and $\varepsilon_{c}$. The values of $e=2 \mathrm{~mm}$ and $s=0.2 \mathrm{~mm}$ lead to $\varepsilon_{c}=2.1 \cdot 10^{-3}, R_{c}=6.1 \mathrm{~mm}$ and $R_{c}=11 \mathrm{~mm}$ at distance $d=0$ and $d=50 \mathrm{~mm}$ respectively.

\subsection{Inorganic scintillating crystal}

The requirements for an inorganic scintillating crystal to be used as the ${ }^{99 \mathrm{~m}} \mathrm{Tc} 140 \mathrm{keV}$ detector, are a high density, high $\mathrm{Z}$ and high light yield. In addition, the crystal should be chosen between the fast decay time crystals in order to be compatible with the use of our fully developed electronics. Table 1 summarizes the available candidates. LSO and LYSO crystals have been rejected because of their lutetium natural radioactivity and afterglow. (The ${ }^{176} \mathrm{Lu}$ emits $\beta^{-}$ 


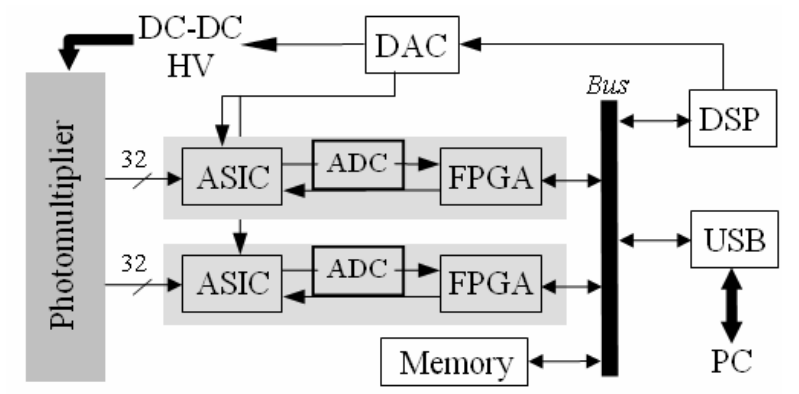

Figure 3. Schematic of the mini Gamma Camera dedicated electronics.

particles at $1.19 \mathrm{MeV}$ with $3.78 \cdot 10^{10}$ of half-life and $\gamma$-rays emissions with a maximum intensity at $88.4 \mathrm{keV}$ ). The best candidate in the different high dense and non hygroscopic crystals, is the cerium doped orthosilicate of gadolinium $\left(\mathrm{Gd}_{2} \mathrm{SiO}_{5}: \mathrm{Ce}\right.$ or $\left.\mathrm{GSO}\right)$. Taking into account its interaction length of $1.7 \mathrm{~mm}$, a thickness of $2 \mathrm{~mm}$ allows $70 \%$ conversion of the $140 \mathrm{keV} \gamma$ rays. Its scintillation wavelength at maximum of the spectra emission, $(\lambda=430 \mathrm{~nm})$, is optimum for the quantum efficiency of a photomultiplier tube bialkali photocathode. In spite of its relatively low scintillation yield, such a crystal enables to propose a compact system with a reduction of the photons spread out reaching the photocathode. In our application, the detection efficiency or the spatial resolution defined as the separation power between close SLN, are more important parameters compared to the energy resolution. The $50 \times 50 \mathrm{~mm}^{2} \mathrm{GSO}$ all polished crystal faces were provided by Hitachi Chemical Co. LTD (Japan). Its entrance surface and edges were covered by $1 \mu \mathrm{m}$ thickness reflective layer of aluminum by the Crytur Company (Turnov, Czech Republic). Moreover, a $1 \mathrm{~mm}$ copper layer is added between the collimator and the crystal entrance surface in order to absorb $56 \%$ of the reemitted fluorescence X-rays produced in the lead collimator.

\subsection{The multianode photomultiplier tube}

The crystal is coupled to a HAMAMATSU H8500 flat panel multianode photomultiplier tube (MAPMT) with a $52 \times 52 \times 28 \mathrm{~mm}^{3}$ dimensional outline and an effective photocathode area of $49 \times 49 \mathrm{~mm}^{2}$. The MAPMT is composed of a $1.5 \mathrm{~mm}$ thick borosilicate glass window, a bialkali photocathode, 12 stages metal dynodes per channel and 64 anode pixels (matrix of $8 \times 8$ pixels). The maximum quantum efficiency of the photocathode is located at a wavelength of $420 \mathrm{~nm}$, well adapted to the $430 \mathrm{~nm}$ emission peak of the GSO scintillating crystal. The anode size is $5.8 \times 5.8 \mathrm{~mm}^{2}$ with a $6.08 \mathrm{~mm}$ pitch. The weight of the MAPMT is $145 \mathrm{~g}$. The working high voltage of $-800 \mathrm{~V}$, has been chosen to obtain a gain of $\sim 10^{6}$, in good agreement with the recommended high voltage given by the manufacturer. In the data sheet provided by HAMAMATSU, the gain dispersion of the 64 channels is 1:3. Due to the fact that a common trigger value is applied to the 64 channels, electrical gain corrections, in a range from 0 to 4 (coded by a 6 bits DAC placed directly in the electronics) are used to make the channels responses uniforms. The assembly of the crystal on the MAPMT window using optical silicone has been performed by the Crytur Company (Turnov, Czech Republic).

\subsection{The electronics}

The dedicated front end electronics [9] developed for this device allows the readout of the MAPMT 64 channels (figure 3). In this way, the signals from all the channels can be more easily calibrated than using a resistive current divider [10]. The 64 signals from the anodes are 


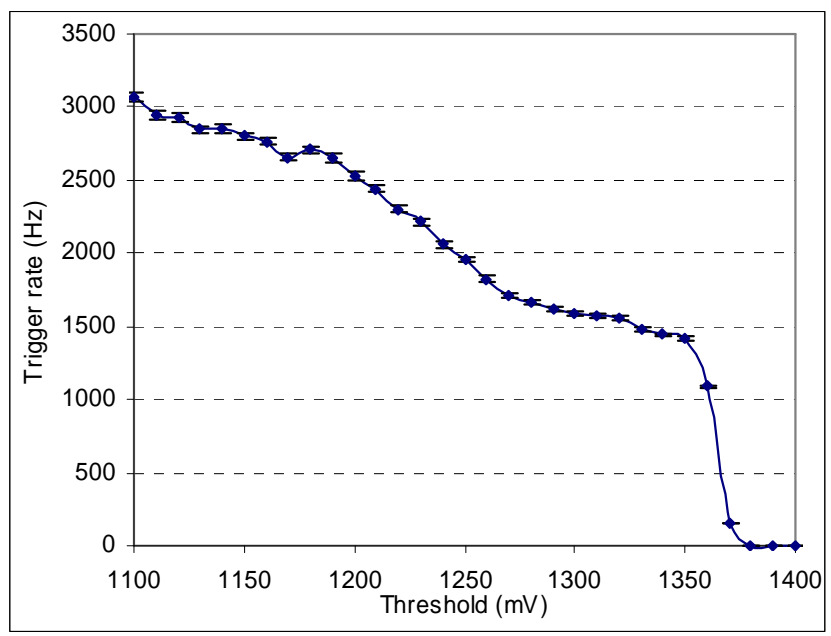

Figure 4. Mini gamma camera trigger rate in $\mathrm{Hz}$ versus the threshold in $\mathrm{mV}$. The red dotted line indicates the chosen working value corresponding to $\sim 3.3$ photoelectrons.

divided in 2 groups (32 channels each) processed in parallel by 2 identical daughter boards plugged into a mother board. For each dedicated daughter board, a Specified Integrated Circuit designed for our Application (ASIC) named OPERA-ROC [11], including gain adjustment, discrimination and shaping, performs triggering and noise signal extracting. The electronic integrates all the necessary components to create digital data from the measured charges and to send them to an acquisition PC through a USB link. Two Field Programmable Gate Arrays (FPGA) drives the segmental signals outputting of the two ASICs and conversion with two Analog to Digital Converters (ADC). A multichannel Digital to Analog Converter (DAC) handles the trigger threshold and the high voltage level. A digital Signal Processor (DSP) controls this electronic and communicates with the acquisition PC. The high voltage is provided by a small DC-DC converter circuit. The trigger is provided by an OR between the two trigger signals from the daughter boards. Each trigger signal is produced in the OPERA-ROC chip and corresponds to the OR of the 32 channels. When at least one pixel is fired, the deposited charges in all the channels are read. The trigger level has been fixed measuring the count rate in presence of a non collimated ${ }^{57} \mathrm{Co}$ source illuminating uniformly the activated gamma camera surface. In figure 4 the trigger rate in counts per second $(\mathrm{Hz})$ has been plotted as a function of the applied threshold. The threshold must be placed in the plateau region beyond the noise count region. Applying this criterion, the threshold value has been fixed to $1320 \mathrm{mV}$. The amplifier gain is $400 \mathrm{mV} /$ photoelectron [11], so, we deduce that our trigger level value is equivalent to $\sim 3.3$ photoelectrons.

\section{Calibration}

To perform the measurements presented in this chapter, the gamma camera was not equipped with its lead collimator. The intrinsic spatial resolution of $3 \mathrm{~mm}$ has been measured using a collimated ${ }^{57}$ Co located placed at $1 \mathrm{~mm}$ distance from the entrance surface of the crystal [12]

\subsection{Energy calibration}

To calibrate in energy each MAPMT channel, a collimated ${ }^{57}$ Co source mounted on an X-Y table, was successively presented in front of each pixel. To situate the $\mathrm{X}-\mathrm{Y}$ table in relation to the 

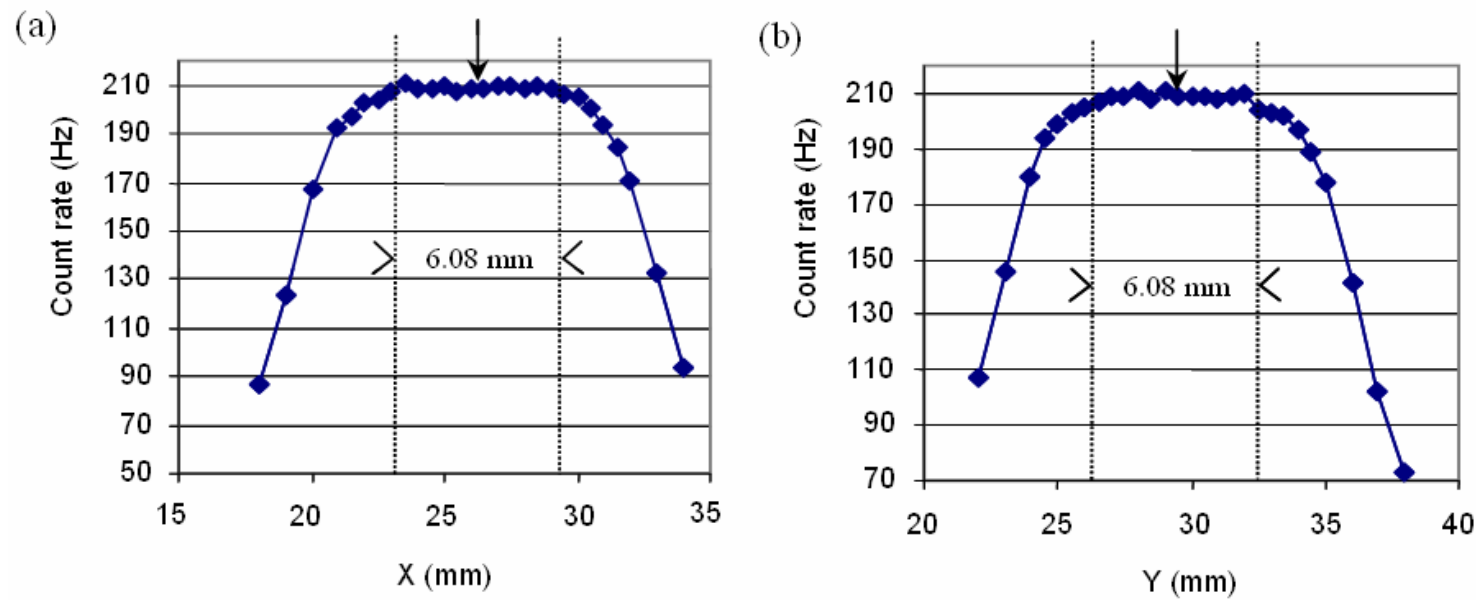

Figure 5. Profiles of the pixel \# 19 in X (a) and Y (b) axis, the dotted lines indicate the pixel size and the arrow indicates its center.

\begin{tabular}{|c|c|c|c|c|c|c|c|}
\hline 1 & 9 & 17 & 25 & 33 & 41 & 49 & 57 \\
\hline 2 & 10 & 18 & 26 & 34 & 42 & 50 & 58 \\
\hline 3 & 11 & 19 & 27 & 35 & 43 & 51 & 59 \\
\hline 4 & 12 & 20 & 28 & 36 & 44 & 52 & 60 \\
\hline 5 & 13 & 21 & 29 & 37 & 45 & 53 & 61 \\
\hline 6 & 14 & 22 & 30 & 38 & 46 & 54 & 62 \\
\hline 7 & 15 & 23 & 31 & 39 & 47 & 55 & 63 \\
\hline 8 & 16 & 24 & 32 & 40 & 48 & 56 & 64 \\
\hline
\end{tabular}

Figure 6. Schematic of the three different areas of the energy calibration with the channels number. In white the central area, in blue the edge area and the corner area in red.

gamma camera position, the determination of the center of three pixels $(\# 19,22,43$; figure 6 ; forming a 90 degrees angle is done by moving the source by steps of $0.5 \mathrm{~mm}$ in two perpendicular directions in front of the pixels. Then, the coordinates of the center of the cell are extracted from the profiles as described in figure 5 for the cell \#19.

The region where the signal ratio is constant $(\sim 8 \mathrm{~mm})$ is larger than the pitch $(6.08 \mathrm{~mm})$ of the MAPMT. This is due to the spreading of the photons in the crystal thickness and their refraction through the MAPMT window glass. Once the three orthogonal pixels centers coordinates known, the collimated source was placed in front of the center of each pixel. For each position of the source, a minimization is applied to the clusters data file in order to calibrate the ADC gaussian distributions to energy distributions (clusters are defined as a group of neighboring fired cells). Then, the energy distributions of the 64 clusters are centered at 


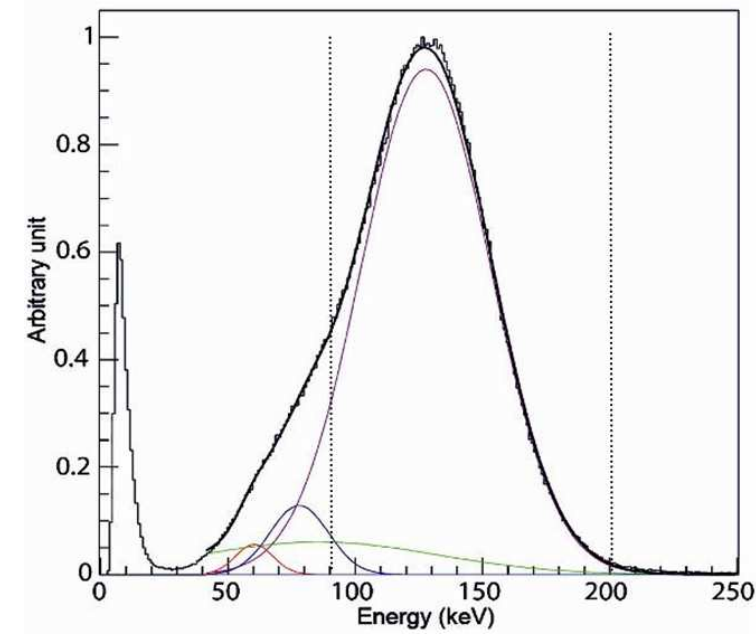

Figure 7. Energy spectrum fitted (black curve) by the sum of Compton contribution (green), tungsten $\mathrm{K} \alpha 1$ ray emission at $59 \mathrm{keV}$ (red), lead $\mathrm{K}_{\alpha 1}$ ray emission at $75 \mathrm{keV}$ (blue) and the $122 \mathrm{keV}{ }^{57} \mathrm{Co} \gamma$ ray (pink). The dashed lines represent the energy window (90 keV to $200 \mathrm{keV}$ ).

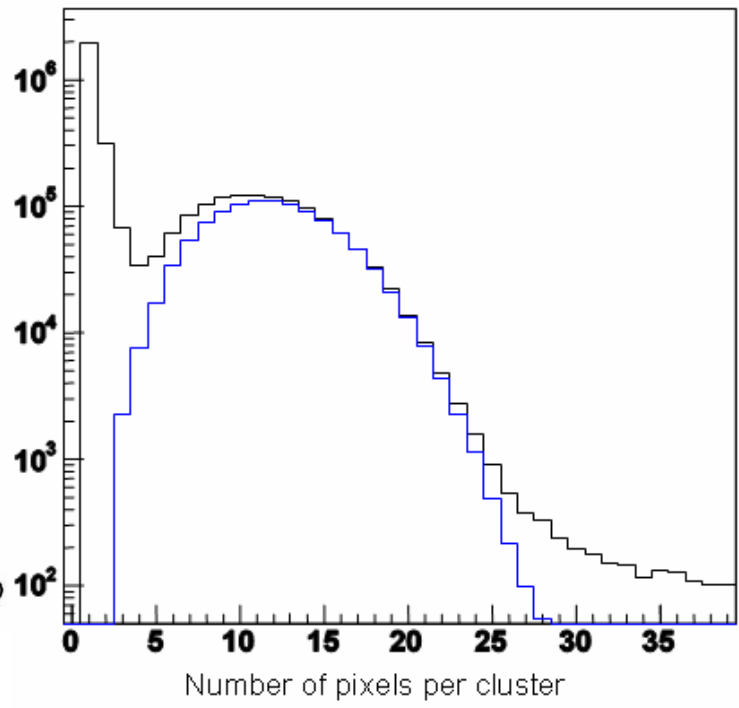

Figure 8. Distribution of the number of pixels per cluster, without cuts (black) and with the cuts defined in section 3.2 (blue).

$122 \mathrm{keV}$ by determination of energy calibration coefficients which are defined into three areas (figure 6). The first area (white) contains the coefficients which have to be applied when events happen in the center of the crystal. The second (blue), when events interact in the edges of crystal and the last (red) for events in the corners. Figure 7 shows the energy spectrum of a sum of clusters for one of the 64 positions, well fitted by the sum of the Compton contribution, the tungsten $59 \mathrm{keV} \mathrm{K}_{\alpha 1}$ ray emission, the lead $75 \mathrm{keV} \mathrm{K}_{\alpha 1}$ ray emission and the ${ }^{57} \mathrm{Co} \gamma$-ray photopeak at $122 \mathrm{keV}$. The dashed lines represent the cuts in energy made on the distribution in order to select the $122 \mathrm{keV}$ contribution. The energy resolution at $122 \mathrm{keV}$ is $47 \%$ $(\mathrm{FWHM}=61 \mathrm{keV})$. The ${ }^{57} \mathrm{Co}$ source emits two different gamma photons at 122 and $137 \mathrm{keV}$ with a proportion of $86 \%$ and $11 \%$ respectively. The last one is not taking into account in the minimization of the energy cluster because it cannot be a priori separate in the cluster energy spectra.

\subsection{The image}

The 64 readout signals are treated event by event using a clusterisation algorithm in order to obtain the image (an event is defined as a signal entering the discriminator with an amplitude greater than the threshold). After the pedestal subtraction, the first step of the treatment consists in the formation of cluster of fired cells, defined as a group of neighboring pixels with a charge greater than an ADC value fixed to 3 times the r.m.s of the pedestal measured distribution. Figure 8 shows the distribution of the number of pixels per cluster obtained with a non collimated ${ }^{57} \mathrm{Co}$ source located at a distance of $40 \mathrm{~cm}$ in front of the entrance surface of the crystal (in black, without energy window cut). In order to avoid considering events that are not produced by the source, the low limit in valid pixels number per cluster is set to 3 (this number corresponds to the lowest number of pixels that can be illuminated by the optical photons from a $\gamma$-ray conversion in the crystal). For the selected clusters, the total energy is given by the sum of the individual pixels contributions. So as to consider only the ${ }^{57}$ Co $\gamma$-rays, an energy window 


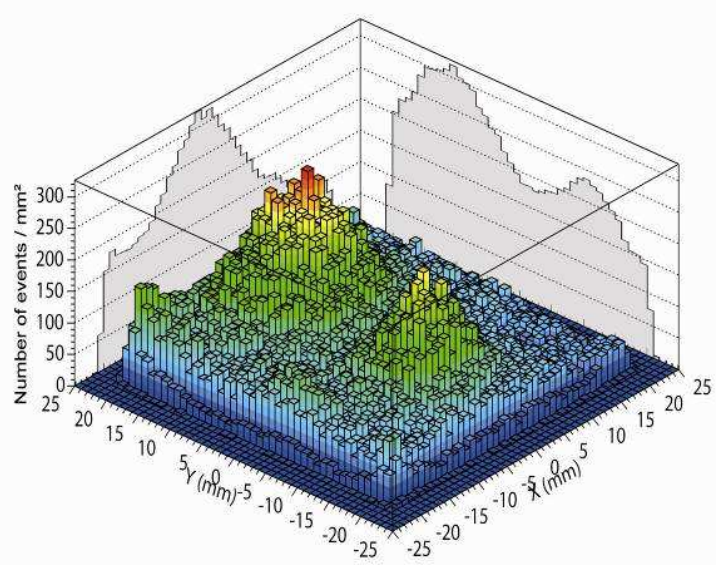

Figure 9. Example of a plot with the profiles (grey) of two ${ }^{57}$ Co phantoms.

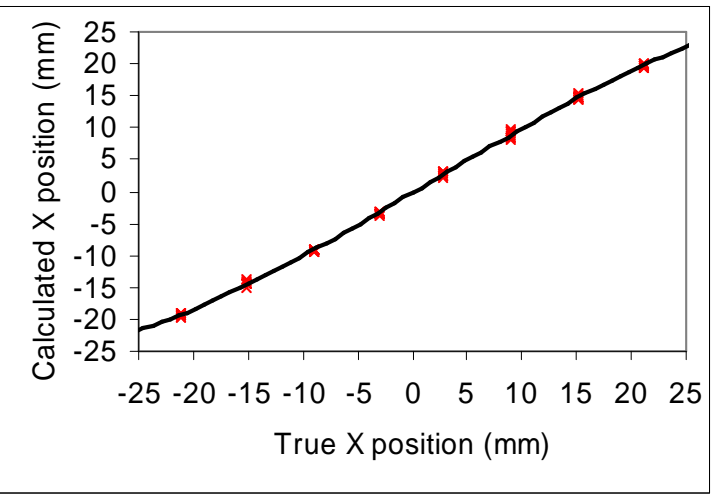

Figure 10. Plot of the 64 pixels calculated positions in the $\mathrm{X}$ direction versus the true $\mathrm{X}$ position of the point-like source. Each red "point" is the superposition of 8 iso-Y positions.

corresponding to $122 \mathrm{keV} \pm_{1 \sigma}^{3 \sigma}$ (figure 7), is applied to the energy spectrum. Then, the mean number of pixels per cluster is equal to $12 \pm 5$ (in blue on figure 8). After all these selections, the $\mathrm{X}$ and $\mathrm{Y}$ positions of the $\gamma$-ray interaction in the crystal are calculated by the weighted centers of gravity of the cluster pixels signal distribution. The different events positions are plotted in a $2 \mathrm{D}$ histogram with a $1 \mathrm{~mm}$ binning in each direction as presented in figure 9 in case of two phantoms image. The cumulative projections along the two axes allow obtaining the two profiles of the histogram.

\subsection{Linearity}

The linearity of a gamma camera is defined as the correlation between the position of the pointlike source of $\gamma$ photons, in front of the crystal entrance surface, and the calculated photon interaction position in the inorganic scintillating crystal.

The collimated source has been first positioned in front of the center of each MAPMT channel. Figure 10 represents the sum of the 8 lines containing the 8 measured positions of the interaction in the crystal $X_{m}(\mathrm{~mm})$ correlated with the real source position $X_{\mathrm{r}}(\mathrm{mm})$. To determine $X_{m}$, a gaussian fit is applied on the profiles of the images. The best fit to the data is given by:

$$
\mathrm{X}_{\mathrm{m}}=-0.0002 \cdot \mathrm{X}_{\mathrm{r}}^{3}+0.0229 \cdot \mathrm{X}_{\mathrm{r}}^{2}+0.1674 \cdot \mathrm{X}_{\mathrm{r}}-25.794 \text {, with an } \mathrm{r}^{2}=0.9994 \text { correlation coefficient. }
$$

At the edges of the crystal, the linearity is not respected. The scintillating photons emitted near the edges would be reflected and then events coordinates will be calculated in wrong weighted interaction positions. We measure a maximum of $10 \%$ deviation from the linearity in this region.

To study in detail this distortion, a more accurate 25 by 25 points scanning with a $2 \mathrm{~mm}$ step in both directions has been performed on the whole crystal surface. The precise knowledge of the real positions of the collimated source allows obtaining $25 \times 25$ correction matrix elements. Figure 11,a,b represent the 1250 calculated interaction positions before and after the application of the linearity correction matrix respectively. 

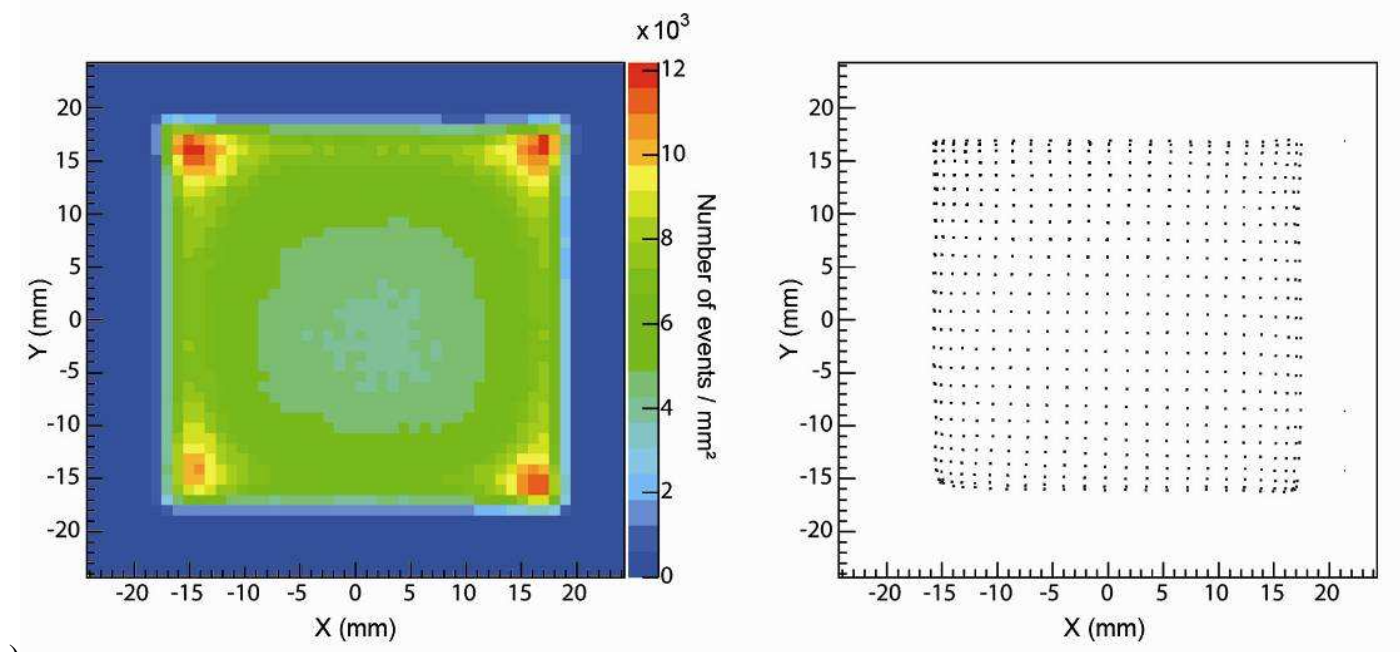

a)
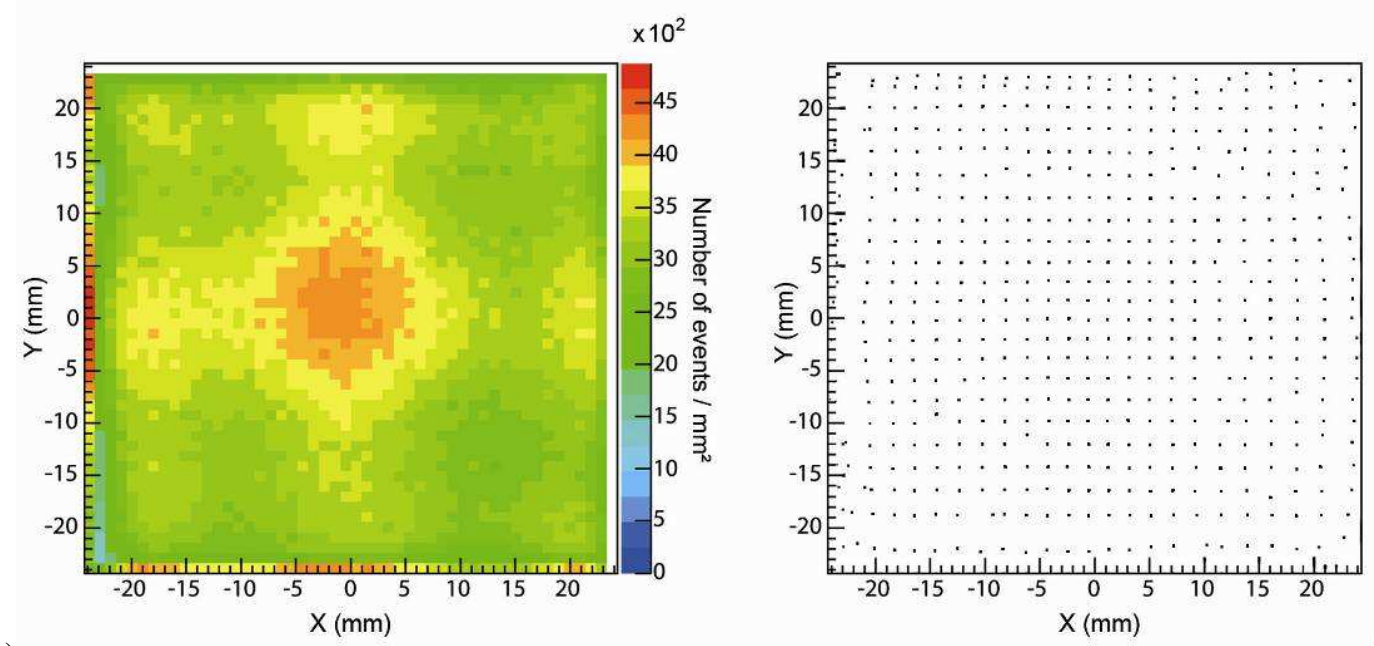

b)

Figure 11. a) Measured positions of the 1250 scanning points before linearity correction represented as the $2 \times 2 \mathrm{~mm}^{2}$ pixel population (left) and their coordinates (right). b) Corrected positions of the 1250 scanning points after application of the linearity matrix coefficients. Note the difference in color codes between the figures $11 \mathrm{a}$ and $11 \mathrm{~b}$.

\subsection{Uniformity}

The homogeneity of the channels responses is first verified by comparison of the number of events collected by each channel during the same data acquisition time, when the collimated source is located in front of. The plot presented in figure 12 a, contents the number of events collected by each channel as a matrix of $8 \times 8$ channels. The data are also presented as the number of recorded events versus the channel number (figure 12b). The number of events collected by the channel 16 is significantly lower than the others. It could come from a default on the crystal at this place (the crystal could have been broken or not well glued on the MAPMT window). However, this first observation proved that the channels respond uniformly.

Nevertheless, to investigate in details the uniformity and the edges effects in the scintillating crystal, a 20 minutes data acquisition with the non collimated ${ }^{57} \mathrm{Co}$ source is performed. The non collimated source is positioned at $40 \mathrm{~cm}$ distance of the collimator to fairly 

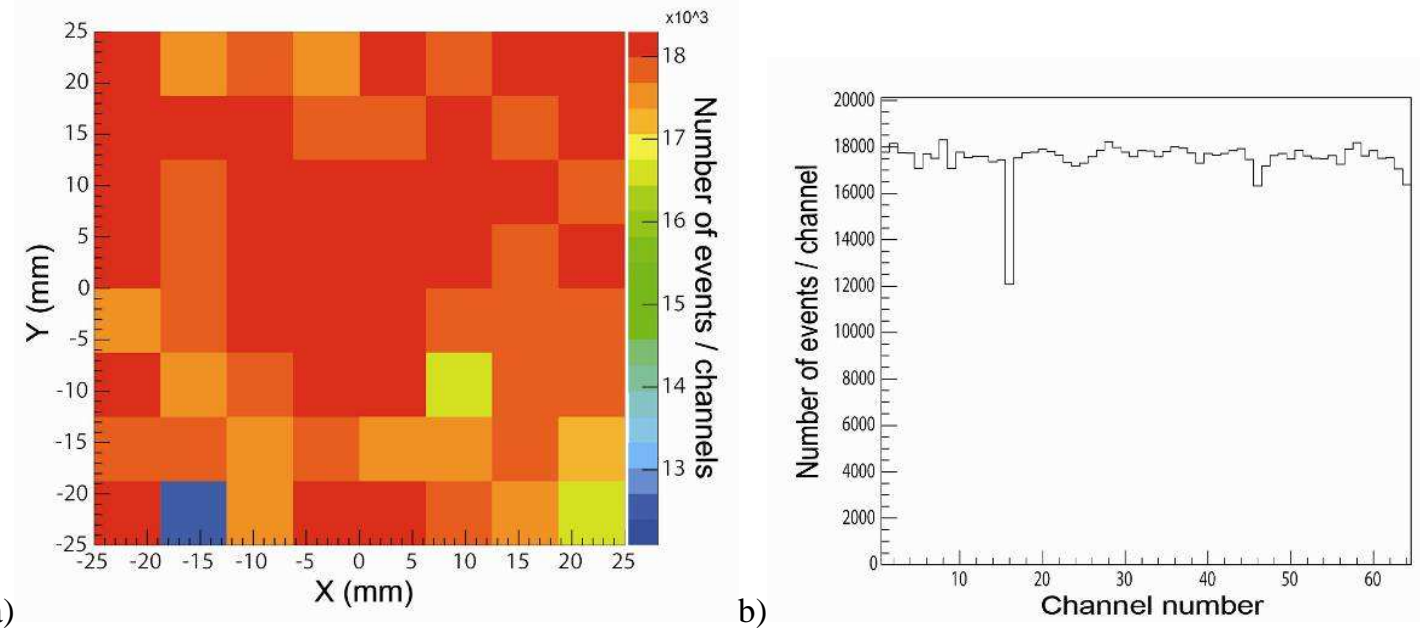

Figure 12. a) Matrix of the $8 \times 8$ channels representing the number of events collected after all cuts when the channel has the maximum energy in the cluster. b) Plot of the number of events collected per channel versus the channel number.

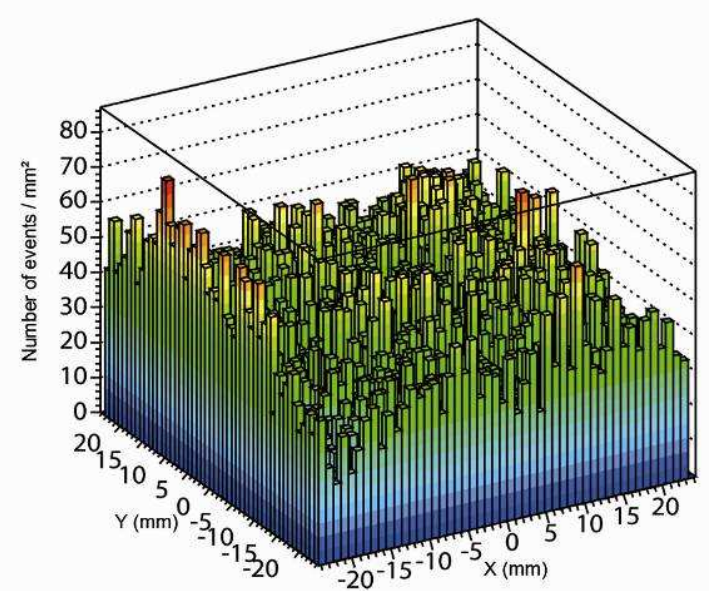

Figure 13. Plot of the "uniform acquisition" before correction.

obtain a uniform $\gamma$ photons flux reaching the crystal of $5 \times 5 \mathrm{~cm}^{2}$. After making the 2D plot as described in section 3.2 (figure 13) representing this "uniform acquisition", the mean count rate value of the $50 \times 50$ image pixels is calculated. This mean value is divided by the content value of each pixel in order to obtain its uniformity correction coefficient. Then, a matrix of 2500 elements is created which has to be applied on the projection pixels contents values.

\section{Phantom study}

The gamma camera equipped with its lead collimator, has been tested using acrylic glass phantoms with similar SLNs activities. Then, the used phantoms activities are ranged from 5 to $30 \mathrm{kBq}$. These phantoms are made by deposing a ${ }^{57} \mathrm{Co}$ solution at the center of an oval acrylic glass of $15 \times 8 \mathrm{~mm}$ axis length.

Figure 14 represents the image of two phantoms of 4.3 and $9.8 \mathrm{kBq}$ positioned in front of the entrance surface of the collimator for a 2 minutes data acquisition. The distance between the 

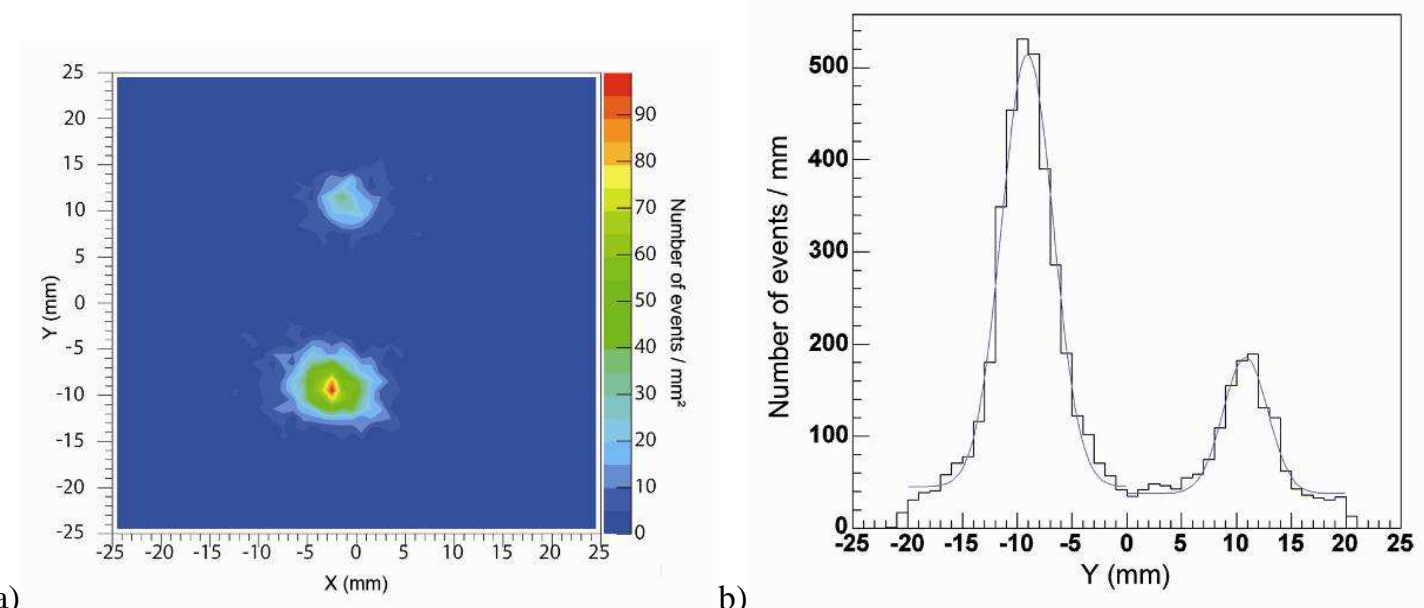

Figure 14. a) Image of two ${ }^{57} \mathrm{Co}$ phantoms of 4.3 and $9.8 \mathrm{kBq}$ separated by $20 \mathrm{~mm}$. b) Y profile of the figure 14a fitted by the sum of two gaussian curves and a flat background (blue curves).

two phantoms is $20 \mathrm{~mm}$. Because of the phantoms central positions in the FOV, the linearity and uniformity corrections are not really relevant.

In a recently published paper [13] we demonstrated the linear correlation between the ${ }^{57} \mathrm{Co}$ phantoms image profiles FWHMs and their distance to the collimator entrance face. On the Y profile presented in the figure 14 obtained from the phantoms image (figure 14 ), the gaussian fits have been applied in order to extract the FWHM of the two phantoms profiles. These values were correlated linearly to the distance to the collimator with the parameters presented in [13]. The values of $\mathrm{FWHM}_{1}=5.9 \mathrm{~mm}, \mathrm{FWHM}_{2}=5.0 \mathrm{~mm}$, are in agreement with the $6.1 \mathrm{~mm}$ linear function intercept, representing the spatial resolution at $d=0 \mathrm{~mm}$. This method is used to give the depth of the SLNs during the preoperative image of the axillary area.

Taking into account that the $2 \mathrm{~mm}$ thick GSO crystal stops $90 \%$ of the $122 \mathrm{keV} \gamma$-rays and that the geometrical efficiency of the collimator is $2.1 \cdot 10^{-3}$, the calculated detection efficiency of this prototype is $2 \mathrm{cps} \cdot \mathrm{kBq}^{-1}$.

Using the three values given by the gaussian fits in the $\mathrm{Y}$ profile, we can extract the number of events collected per second by the gamma camera $(30.9 \pm 0.6 \mathrm{cps})$. The total activity emitted by the two phantoms is equal to $14.1 \pm 0.1 \mathrm{kBq}$. Then, the detection efficiency is $2.19 \pm 0.05 \mathrm{cps} \cdot \mathrm{kBq}^{-1}$ in good agreement with the previous theoretical calculation.

\section{Conclusions and perspectives}

We presented a new intra-operative gamma camera that combines high detection efficiency $\left(2 \mathrm{cps} \cdot \mathrm{kBq}^{-1}\right)$ and a good spatial resolution (10 $\mathrm{mm}$ for a $50 \mathrm{~mm}$ distance to the collimator). The results presented here were obtained for a prototype that represents one quarter of the final FOV gamma camera design. This camera has been evaluated during a clinical trial with a series of 25 patients at the Hôpitaux Universitaires de Strasbourg [14]. It has been installed on a mechanical support to produce the postoperative scintigraphic images in order to check the complete SLNs clearance (figure 15). These images can be used as a quality control documents.

The images are preformed in $2 \mathrm{~min}$. data acquisition time. However, because of its reduce FOV, the camera has to be moved to obtain four images in order to cover the whole surface of the axillary area, with possible recovery. The time of acquisition is then multiply by four. 


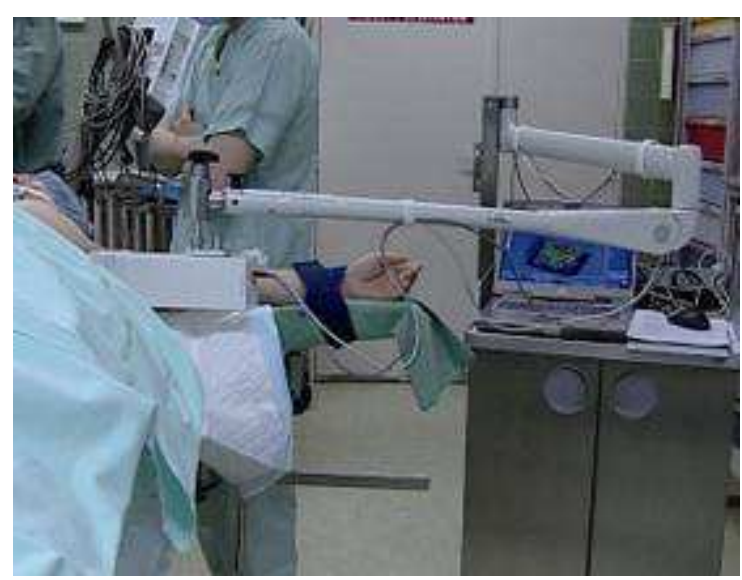

Figure 15. Picture of the mini gamma camera on its support.

The next step will introduce the new design of the gamma camera with a FOV of $100 \times 100 \mathrm{~mm}^{2}$. This new prototype will be declined in two different configurations. The first will use four GSO:Ce crystals of $50 \times 50 \times 2 \mathrm{~mm}^{3}$ each coupled to four MAPMTs H8500. The second one will use an unique $100 \times 100 \times 2 \mathrm{~mm}^{3}$ GSO:Ce crystal glued to four H8500 MAPMTs. The performances of these two prototypes will be evaluated and compared on 100 patients in a new clinical trial during autumn 2007.

\section{Acknowledgments}

We thank Professor Jean-Philippe Brettes for his interest in the CarolIReS gamma camera development and David Brasse for his helpful discussion.

This work was supported by funds of the Comités du Haut-Rhin et du Bas-Rhin de la Ligue National contre le Cancer.

The authors gratefully acknowledge the Région Alsace for its support.

\section{References}

[1] G.H. Lyman et al., American Society of Clinical Oncology guideline recommendations for sentinel lymph node biopsy in early-stage breast cancer, J. Clin. Oncol. 23 (2005) 7703.

[2] A. Abe et al., Performance evaluation of a hand-held, semiconductor (CdZnTe)-based gamma camera, Eur. J. Nucl. Med. Mol. Imaging 30 (2003) 805

[3] I.M. Blevis et al., Evaluation of a CZT intra-operative Gamma Camera, IEEE Nucl. Sci. Symp. Conf. Rec. 6 (2004) 3555

[4] D. Kopelman et al., A newly developed intra-operative gamma camera: performance characteristics in a laboratory phantom study, Eur. J. Nucl. Med. Mol. Imaging 32 (2005) 1217

[5] M. Tsuchimochi et al., A prototype small CdTe gamma camera for radioguided surgery and other imaging applications, Eur. J. Nucl. Med. Mol. Imaging 30 (2003) 1605.

[6] S. Pitre, L. Ménard, M. Ricard, M. Solal, J.R. Garbay and Y.A. Charon, A hand-held imaging probe for radio-guided surgery: physical performance and preliminary clinical experience, Eur. J. Nucl. Med. Mol. Imaging 30 (2003) 339. 
[7] J.N. Aarsvold et al., Gamma cameras for intraoperative localization of sentinel nodes: technical requirements identified through operating room experience, IEEE Nucl. Sci. Symp. Conf. Rec. 2 (2002) 1172

[8] C. Mathelin, I. Piqueras and J.L. Guyonnet, Performances of a new prototype handheld scintillating probe for the search of sentinel lymph nodes in breast cancer, Eur. J. Surg. Oncol. 32 (2006) 24

[9] P. Bard et al., Electronics dedicated to multi-anode photomultiplier tubes for imaging scintigraphic devices, $14^{\text {th }}$ IEEE-NPSS Real Time Conference, Stockholm, June 4-10, 2005.

[10] S. Siegel et al, Simple charge division readout for imaging scintillator arrays using a multi-channel PMT, EEEE Trans. Nucl. Sci. 43 (1996) 1634.

[11] A. Lucotte et al, A front end read out chip for the OPERA scintillator tracker, Nucl. Instrum. Meth. A 521 (2004) 378.

[12] I. Piqueras, B. Gizard, D. Brasse, C. Mathelin and J.L. Guyonnet, A new mini gamma camera for sentinel lymph node identification in patients with breast cancer, SCINT Alushta, Crimea, Ukraine, September 19-23, 2005.

[13] C. Mathelin, S. Salvador, D. Huss and J.L. Guyonnet, Precise localization of sentinel lymph nodes and estimation of their depth using an intraoperative gamma camera in patients with breast cancer, J. Nucl. Med. 48 (2007) 623

[14] http://www.clinicaltrials.gov/ct/show/NCT00357487. 